\title{
A Review of Secretion Systems in Pathogenic and Non-Pathogenic Bacteria
}

\author{
Elaheh Gholami Parizad ${ }^{1}$, Eskandar Gholami Parizad ${ }^{2}$, \\ Iraj Pakzad ${ }^{3}$ and Azar Valizadeh ${ }^{1}$
}

\author{
${ }^{1}$ Clinical Microbiology Research Center, Ilam University of Medical Sciences, Ilam, Iran \\ Student research committee, Ilam University of Medical Sciences, Ilam, Iran \\ ${ }^{2}$ Psychosocial Injuries Prevention Research Center, Ilam University of Medical Sciences, Ilam, Iran- \\ ${ }^{3}$ Department of Public Health, Ilam University of Medical Sciences, Ilam, Iran.
}

http://dx.doi.org/10.13005/bbra/2016

(Received: 29 December 2016; accepted: 15 Februay 2016)

\begin{abstract}
Secretion is an essential duty for prokaryotes to better interact with their surroundings or host. In particular, the production of extracellular proteins and peptides is important in many aspects of survival and organism adaptation to its ecological niche. Secretion systems in bacteria are multi component protein structures used to transport molecules across bacterial membranes. Secretion systems are usually classified into 7 groups: Type I, II, III, IV, V, VI, VI and chaperons navigating the pathways are also a part of the system. Since this systems can transport a variety of different pathogenic factors outside of the bacterial cell and establish the ability to communicate with the environment in which the bacteria live. This is important about the human pathogenic bacteria because by understanding the components and functions of these protein transport systems, we can find the right solution to deal with them. Furthermore, these systems can be used for biological fights.
\end{abstract}

Keyword: secretion systems, sec system, Tat system, protein traffic, transfer proteins.

\section{Background}

Bacteria use different mechanisms to interact with the external environment or host. Most of these mechanisms are based on production and secretion of proteins. The process of protein secretion in bacteria has different and sometimes complex mechanisms and depends on the structure of bacteria. Accordingly, this process will be distinct in positive and negative gram bacteria due to differences in the structure of the walls and the bacterial cell membrane.

S atement of the problem

Despite much research and significant ad vances in understanding the secretion systems,

To whom all correspondence should be addressed. Te l: +98 84 32240404; Fax:+ 988432240404

E-nail: Eskandar-parizad@yahoo.com procedure and structural and molecular mechanisms of these systems are not clearly specified. In some bacteria, such as Mycobacterium (tuberculosis agent), the use of secretion systems to cause virulence is very important. Therefore, if these systems be at service of the bacteria as a virulence factor, the importance of accurate and complete understanding of the components and their performance will increase.

\section{Objective of research}

Given the importance of bacterial systems in pathogenicity of organisms (human, animal, and plant), more understanding these systems can promise solutions to deal with them. Moreover, in an ideal landscape, access routes to the effective and targeted drugs or even using them in biological fights is not unexpected. 


\section{DISCUSSION}

In Gram-negative bacteria, 6 secretion systems are known and named as Type I to Type VI. Each system has its own different components, compounds and the mechanisms. In these bacteria, materials must pass through both the inner and outer membranes or certain substances should enter into the host cell and therefore various molecules and mechanisms are required for this purpose. Gram-positive bacteria are common by Gram-negative bacteria in some secretion systems and pathways; although, most of them benefit from $\mathrm{Sec}$ and Tat secretion pathways to discharge materials through the single-layer membrane width $^{1}$. Systems I, III, IV, VI are single-stage pathways. This means that the materials they carry are discharged into the extracellular space directly and without any periplasmic intermediate (2). In Gram-positive bacteria with single-layer membrane, single-stage systems and system VII can be found in Gram-positive bacteria with unusual walls, such as mycobacterium, and are special for these bacteria. Number of System T7SS stages (onestage or two-stage system) is not clearly known yet ${ }^{1-3}$.In two-stage systems such as II and V, proteins with the help of a general secretion systems such as Tat and Sec enter into periplasma space to find the right folding, and then in the second step, protein finds the way out by one of the two-stage secretion systems ${ }^{4}$.

While several secretion systems dedicated in Gram-negative bacteria have evolved to facilitate transportation processes, in intracellular bacteria lacking an outer membrane such as cellular walls without mollicutes and Grampositive bacteria such as: Listeria monocytogenes and Rhodococcus equi, materials can easily be transported to outside of cell without using sophisticated equipment $t^{5-7}$.

\section{Type I secretion systems(T1SS) or ATP-binding} cassette(ABC) transporters

This is a single-stage system that directly leads proteins to outside of the cell membrane. In this course, no processing is done on proteins and periplasmic intermediation does not occur ${ }^{1}$. Many proteins are secreted by this system, such as Hemolysins and Loco Toxins that play an important role in the pathogenesis of bacteria.
Moreover, bacteriocins produced by bacteria against other species, find way out by this system ${ }^{2}$ 6-8.

Other proteins secreted by this system, include those that play role in the absorption of nutrients such as proteases, lipases and also iron scavenger proteins which are called HasA. Some of the proteins and compounds such as exopolysaccharides involved in the formation of biofilm are removed by this system from bacterial cells ${ }^{2,7}$.

\section{Components of Type I secretion system}

Type I secretion system (T1SS) is composed of three major components: ATPbinding cassette (ABC), Outer Membrane Factors (OMFs), Membrane Fusion Protein) MFP ${ }^{2,6}$.

Applying ATP hydrolysis, the energy required for Type I secretion system is provided and other parts extend the protein secretion system in the outer/inner membrane. Structurally, OMF provides a channel that penetrates from periplasm to outer membrane and leads the materials from periplasm to outer membrane. In fact, OMF forms a hole through the outer membrane and finally MFP as an anchor on the one hand is connected to the inner membrane and covers periplasmic space and on the other is connected to the large periplasmic domain of the outer membrane $\mathrm{OMF}^{9,10}$.

In fact, MFP is responsible for communication between the OMF and ABC transport system in periplasmic space. Such a system can be found in Gram-positive/negative bacteria ${ }^{11}$. Proteins that contribute $A B C$ system components in T1SS can be classified in two groups:

A group dedicated to withdraw large proteins in Gram-negative bacteria and the other for exporting peptides and small proteins. ABC system in T1SS has two cytoplasmic regions for ATP hydrolysis and two regions passing through the membrane (trans-membrane). ABC transporter phylogeny reflects its context specificity that rarely undergone any change during evolution ${ }^{10-11}$.

Components of ABC transporter of the T1SSfor HasA and HlyA Serratia and E.coli, are MFPs, OMFs, HasD/HasE/HasF and HlyB/ HlyD/TolC, respectively ${ }^{12-13}$. In human E. coli uropathogenic strain, there is a hemolytic toxin called HlyA ${ }^{12,13}$. 
TolC is one of the trans-membrane proteins (integral)located on the outer membrane while $H l y D$ (MFP)and HlyB (ABC) $)^{7,8}$ occupy preplasmic space and the inner membrane. It is suggested that HlyA should be secreted as an unfolded peptide with a method depended on Gro$E L^{9,10}$.

Compositions of the outer membrane enjoy greater specificity to carry their substrate but OMFs participate in multiple transport processes and prevent from working specifically much. For example, TolC (OMF) of E. coli bacteria is involved not only in HlyA release, but also in releasing colicine $V$ and $M c c V^{14}$, it plays role in leaving toxic substances and resistance to antibiotics as well ${ }^{15}$.

Virulence factors, such as metalloproteinase, adhesions and secretion of glucanases via Type I secretion system can be found in plant pathogens as follows: Agrobacterium tumefaciens, Pseudomonas syringae, Ralstonia solanacearum, Xanthomonas axonopodiscitri and Xylella fastidiosa, Mesorhizobium loti and Bradyrhizobium japonicum ${ }^{16}$.

\section{Type II secretion system (T2SS)}

In Gram-negative bacteria, Type II secretion system is one of the five secretion systems that allow exporting proteins within the bacterial cell to outside or the target host cell ${ }^{17-19}$. Also, studies have shown that TIISS can increase virulence in humans, animals and plants ${ }^{17}$.

Type II secretion system is a two-step pathway in which the protein with the help of Sec and Tat systems pass across the inner membrane and after a short period, are transferred into the extracellular space of bacteria ${ }^{18,21}$. All proteins secreted by this system have a signal sequence at $\mathrm{N}$-terminal end targeted for main terminal branch ${ }^{5}$, 22 .

Klebsiella, Pseudomonas, Vibrio and Aeromonas are examples that offer a good understanding of the mechanism of Type II secretion system. It is also specified that this system has an evolutionary relationship with Type IV Pilli ${ }^{22}$.

Components of Type II secretion system (T2SS)

Genotypic and phenotypic analysis of 12 different bacterial genera identified that this system has 12 components:
Secretion component is effective on the outer membrane (T2S D), a cytoplasmic ATPase (T2S E), carrier protein membrane (T2S F), major Quasi Pilin (T2S G)and Minor Quasi Pilin (T2S H, I, $\mathrm{J}, \mathrm{K}$ ) facilitators of ATPase connection to inner membrane along with $\mathrm{T} 2 \mathrm{~F}$, components that have the substrates of transport in internal membrane (T2S L, M), peptidase quasi-pilin precursors/methyl transferase (T2S O) that also act on pilin Type 4 and a protein that may help identify substrate and secretion interactions (T2S C) ) $^{20-22}$.

The subunits of this system are named from E.coli GSPA to GSPO ${ }^{22-24}$. Evidence suggests that once the proteins remain in Periplasm, they are located in a quasi-endemic state with the help of certain chaperon slike $D s b A^{23}$.This stage of folding is necessary for crossing the outer membrane width. TIISS genes exist in most human and plant pathogenic Gram-negative bacteria ${ }^{20}$. This system is very common in G-Proteobacteria ${ }^{20-}$ ${ }^{22}$.Fifteen types that act as representatives for the presence of genes in this type of secretion system include:

Acinetobacter, Aeromonas, Erwinia, Escherichia, Idiomarina, Klebsiella, Legionella methylococcus, Photobacterium, Pseudomonas, Shewanella, Vibrio, Xanthomonas, Xylellaand Yersinia. Moreover, It is known that TIISS exists among a-Proteobacterias such as Bradyrhizobium, Caulobacter, Gluconacetobacter, Mesorhizobium and b-Proteobacteria such as Azoarcus, Burkholderia, Chromobacterium, Ralstonia and $d$-Proteobacteriassuch as Bedellovibrio, bacteriovorus, Geobactersulfurreducens ${ }^{20,23,24}$.

The types of proteins that are secreted by this system include Acyl Transfrar, amylase, Cellulase, pectinase, chitinase, ADPRibosylenzymes, proteases, lipases, phospholipases A and C, Isophospholipases, alkaline phosphatase, nuclease. It has been seen that in Legionella pneumophila bacteria, mutation in the gene T2S F makes the bacteria unable to survive in mouse's lung cells ${ }^{22}$.

The role of Type II secretion system in pathogenicity

This system is present in developing many plant and human and animal pathogens. It is suggested that this system can be involved in the development of tissue and cell damage of the host. For example an enzyme secreted by this system 
severely helps virulence.

Other cases can be toxin ADP-Ribosyl enzym in E.Coli, cholera toxin, Vibrio cholera and exotoxin A, Pseudomonas aerogenusa ${ }^{15,16}$.

In general, pathogens that express TIISS cause many diseases. Range of disease in humans is extended from pneumonia (L. pneumophila, $P$. aeruginosa) to urinary tract infection (E. coli)and diarrhea (V. cholera) ${ }^{20}$.

\section{The role of Type II secretion system in} environmental niche

Recent studies show that Type II secretion system in plants leads to their growth in the environmental niches. Genetic and functional analyses on 16 non-pathogenic bacteria found in the environment show that these bacteria live freely in soil and water (e.g. A.calcoaceticus, S.oneidensis) to commensal life with plants, (e.g. P. fluorescens) to synbiotic life with plants (e.g. $B$. japonicum)and animals (e.g. V.fischeri).

It was recently shown that TIISS may facilitate intracellular growth in fresh water amoebas in L. peneumophila and cause extracellular growth of bacteria at low temperatures $\left(12-258^{\circ} \mathrm{C}\right)^{25}$.

Several configurations are suggested for Type II secretion system (TIISS)but it is certain that strings and B-barrely structures in the external membrane, constitute 12-14 subunits of secretion system complex ${ }^{22-24,26}$.

Evidence show that probably protein E in the process of secretion with energy supply and assembly of $\mathrm{G}$ and $\mathrm{K}$ quasi Pilin its acts as a kinase regulator. No interaction was detected for Protein $\mathrm{F}^{22}$.

G \& K proteins have homology to Type IV pilin protein $\mathrm{k}$ and it is believed that they form psuedopilus $^{17,18}$. Processing in the N-terminal region is methylation which is carried out by a prepilin peptidase called protein $\mathrm{O}^{1,5}$ and 6 . It was found that a protein called $\mathrm{S}$ also plays role in stability of Protein in the outer membrane ${ }^{23}$.

\section{Type III secretion system(TTSS)}

Type III secretion system is seen in Gramnegative bacteria that can interact with both plants and animals, or they are pathogens and have mutualistic life ${ }^{1}$ to be activated, they need contact with their host cell. Moving in this system occurs in a single-stage and dependent on a structure called injectisom, whether in the construction of this structure, and whether in the displacement of proteins is related to ATP hydrolysis and in its absence is related to $\mathrm{PMF}^{27-29}$.

Type III secretion system was first found in bacteria in the roots of the nitrogen-fixer plants but later it became clear that this system plays an important role in the virulence of pathogenic bacteria as well. Type III secretion system was found in Yersinia species for Yop protein secretion for the first time ${ }^{26}$. Since then, the system was detected in mammalian and plant's pathogens such as: Salmonella eneterica, Shigella flexneri, E. coli, Ralstonia solancearum, Pseudomonas syringae and Chlamydia trachomatis ${ }^{27}$.

Coding genes of the Type III secretion system are located on a chromosomal or plasmid locus and these genes are conserved in different species $^{26}$. Like the System TOSS, TTSS pass its effective molecules across internal and external membrane width independently from system Sec. Mechanism of these effective molecules is still under debate ${ }^{17-19}$. The machine of this system is a structure known as Injectisom ${ }^{27}$.

Multiple Type III secretion system in bacteria such as Burkholderia pseudomallei and its associated species have been identified and are a combination of the Type III secretion systems classified as TTS1, TTS2 and are also shown with symbol TTS3 or Bsa. Type III secretion systems, i.e., (TTS2) and (TTS3) both can be seen in bacteria B. pseudomallei and B. mallei. While, TTS1 is specifically more effective for pathogenisis of $B$. pseudomallei strains ${ }^{26-29}$.

\section{Components of Type III secretion system}

The most important component of Type III secretion system is injectisome which is described below:

\section{Injectisome structure}

Although over 25 proteins are required to assemble the injectisom construction but only 9 of them is protected in 7 families of Type III secretion system. It seems that this structure to have shared evolutionary origins with flagellum. It is known that, this structure is much similar to protein pilus EspA in E.coli. ProteinsFli H, I, J, O, $\mathrm{P}, \mathrm{Q}, \mathrm{R}$, FlhA, B, form the inner membrane structure and the basal body ring and cytoplasmic part of injectisom ${ }^{27}$. The main function of injectisom is to deliver effectors proteins from bacteria and host cell membrane into the cytosol of host cells where they may disrupt the host cell functions through 
various types such as immune and defensive responses ${ }^{27,30}$.

Injectisom structure is studied in animal pathogens such as Yersinia pestis and Salmonella typhimurium as well as plant pathogens such as Pseudomonas syringae. Injectisom consists of a series of basal rings: Fli H, I, J that connect the inner and outer bacterial membrane to a hollow needle (FliO, P, Q, R, FhA, B in Yersinia) or to the filament (in Salmonella) or to Pilus (P. syringae). Each of these structures creates a hole within the cell plasma membrane that can transfer proteins in the cytosol or the cytoplasm ${ }^{17,18,27,30}$. A protected ATPase is along with injectisom structure to provide the energy required to move. Two classes of chaperons play role in assembly of injectisom structure, while the three classes of them are involved in secreting proteins in the secretion process ${ }^{27}$.

In plant pathogenic bacteria, the genes that code TIIISS are known as hypersensitive response ( $h r c)$ and pathogenicity because mutation in these genes distracts bacterial potential for pathogenicity in plant and non-plant host ${ }^{29}$.

The Type III secretion systems in the environmental niche

One of important information researched about the Type III secretion system is that this system is not limited only to pathogens but it exists in commensal and symbiotic bacteria as well.

The first clue of this discovery was to find mega-plasmid that encodes this system in Rhizobium species. NGR234 plasmid gives the ability to use this excretory system to bacteria coexisted with leguminous plants ${ }^{19}$.

Now, three animal bacterial endo symbiont have been shown that are likely to have Type III secretion system. The first case is the bacteria Photorhabdus luminescens which is endosymbiont in insect pathogenic nematodes which probably have a system similar with YscJupp. The second case is Sodalis glossinidius bacteria that have an endosymbiotic life in Glossina (tsetse flies) and enjoy a Spi-1-like system for invasion ${ }^{27}$. It is also believed that the TTSS is located among clustered genes that encode serine/ threonine protein kinas (CT664 in the Chlamydia terachomatis) ${ }^{28-30}$.

Type IV Secretion system $=$ TFSS

Compared to other secretion systems,
Type IV secretion system (T4SS)is unique for its ability to transfer nucleic acids and proteins in plant and animal cells as well as in yeasts and other bacteria $^{31-34}$.

This secretion system is seen both in Gram-negative and Gram-positive bacteria.This mechanism is also applied in the process of a bacterial conjugative plasmid from a bacterium to another one.

In addition, creating antibiotic resistance and contagious genes can lead to big new and important problems ${ }^{17,18,34}$.

Agrobacterium tumefaciensC58 (VirB), Helicobacter pylori (CAG; ComB), Pseudomonas aeruginosa (TraS/TraB), Bordetella pertussis (Ptl), E. coli (Tra), Legionella pneumophila (Dot) have the similar type IV secretion system.

\section{Classification of Type IVA Secretion system}

Type IV secretion system is divided into several groups. In some species, T4SSs similar to Agrobacterium tumefaciens (VirB/VirD4 T4SS) are called as IVAO secretion systems, while T4SSssimilar to Legionella pneumophila Dot/Icm T4SS are commonly known as type IVB secretion systems $^{35,36}$. T4SSs that are located in none of these classes are not usually named as Other $\mathrm{T}_{4} \mathrm{SSs}^{36}$. A large group of "Other T4SSs"include other types, which consist of G1 T4SSs coded by a number of genomic islands, such as clc, pKLC102 and PAPI from Pseudomonas, SPI-7 from Salmonella and also a group of integrated conjugation elements (ICE) from Haemophilus ${ }^{34}$.

Type IVA secretion systems, like VirB/ VirD4 T4SS that are responsible for transmitting A. tumefaciensgenes in cells located in host are considered as early T4SS. A. tumefaciensvirB/virD T4SSrecoded by $12 \mathrm{Ti}$ plasmids open reading frames (virB1-virB11 and virD4).

11 frames out of 12 open readable frames A.tumefaciens virB/virD T4SS are classified in (virB and virD) unit operons, while $\operatorname{VirD4}$ is placed separately. Type IVA secretion systems'pilus is made of pilin subunits VirB2 and VirB5located at the tip of Pilus. Transmission channel of developing-expanding cell is made by encoded proteinsVirB6-VirB10 $0^{37}$.

Small inner membrane which is concerned to protein $\operatorname{VirB3}$ is sustained by $\operatorname{VirB4}$, VirB7 and VirB8 and used in pilus biogenesis ${ }^{6,37}$. VirB4, VirB11 and VirD4 ATPase provide required energy 
for Pilus biogenesis and transporting sub-material through T4SS channel.VirD4 makes conjugator protein double to emphasize on its imminent impact in recognizing and using the substrate in the T4SS secretion channel ${ }^{6,32,37}$.

A number of toxic genes resistant to antibiotics and dependent on catabolism intensify virulence and general bacterial compliance through transporting materials by T4SS. This process involves obtaining ICE antibiotic resistant genes, virulence and dependent on catabolism in Haemophilus and Pseudomonas ${ }^{33,34}$.

Transmission mechanism of Type IV secretion systems

To investigate T4SS, Agrobacterium tumefaciens C58 is used as the best model to describe the system. VirB system of Agrobacterium tumefaciens $C 58$ is able to bring out the proteinDNA complex host Ti plasmid DNA-protein. The main mechanism of pathogenesis is T-DNA injection to host in order to stimulate the cancerous growth or creation of crown-shaped blistering tumors which then produces carbon and energy sources for pathogens. The main components of T4SS in Agrobacterium tumefaciens areC58, VirB2-VirB11 and VirD4. VirB1is responsible for the re-modeling of peptidoglycan using lytic activity of transglycosylase. A major portion of proteins $\operatorname{Vir} B$ is responsible for the formation of complex structure of secretion mechanism that is fed through ATP hydrolysis. VirD2 sends T-DNA into the plant host cell and, plant nuclease 3 also plays a role in this process ${ }^{1,32}$. Protein(s) involved in the transmission affairs, play role in processing DNA in the conjugal transmission source (oriT) to form conjugation intermediates.

Thus, Type IV DNA delivery systems are very similar to mechanisms of protein secretion, which are together with transporting the feeding substrate recognition signals and a piloting function to manage loads, covalent border ssDNA, are transmitted through the channel ${ }^{33}$. Recent researches on conjugation systems show that the transfer of proteins can be done independently from DNA in the receptor cells too. In the method of transferring T-DNA Agrobacterium tumefaciens, VirE2 transmits a sSDNA-binding (SSB) protein and $\operatorname{VirF}$ which is another virulence factor. Other Genetic studies showed that the protein substrate and conjugationinterfaces compete together to reach transfer machines T-DNA 3 and 9.Ptl system in $B$. pertussis that releases pertussis toxin (PT) also benefits from this type of secretion mechanism ${ }^{32,35}$. It should be noted that the path for transmitting PT is an exception unlike other Type IV systems, Pertussis toxin is secreted to surrounding exterior cells, rather than being directly transmitted to a host cell ${ }^{22,35}$.

Several pathogens exist in mammals that are in need of this type of secretion system. For example, in system L. pneumophilaDot / Icm any mutation in genes of this secretion system leads to loss of function of dot/icm by which the wrong bacterial targeting in intracellular division or Lysosomal occurs ${ }^{32,35}$.

\section{Type V Secretion system=T5SS (auto transporters)}

Perhaps the simplest mechanisms of protein secretion are the ways classified in the group of Type V secretion system. This is a twostage system and dependent on $\mathrm{Sec}$ and has a structure similar to beta barely. Moreover, this system also needs to multiple signal peptides. In this type of system, transport includes: Auto transporter system (type Va or AT-1), the TwoPartner Secretion method (type Vb), and a recently described type Vc system (and also AT-2 method). The secreted proteins by these methods are very similar in terms of basic structure as well as biogenetic states ${ }^{18,17,26}$. Genomic sequence shows that automatic transporters are always present among the Proteobacteria. This system's genes are transferred horizontally. Transfer of genes between far apart tissues rarely occurs. Analysis of the current PSI-BLAST databases (unpublished database IR Henderson) shows that the species that use automatic transmission system use more than 700 members, so that the greatest secretion system can be found in Gram-negative bacteria. There are several reports that prove the existence of automatic transmitter (TSSV) outside Proteobacteriae especially in the large family of polymorphic proteins in Chlamydia species. Besides, there is at least a well-known cyanobacterial automatic transmitter ${ }^{22}$.

In analyzing genomic sequence, the number of automatic transporter proteins in most genomes, including Brucellaabortus, Bartonella, Pseudomonas and E. coli is indicated. More comprehensive analysis of genomes $P$. aeruginosa 
and $P$. fluorescens, show the presence of three and nine automatic transporter proteins. The comparison of several bacterial pathogen genomes 'distribution of multiple automatic transporters is similar to each other and to automatic transporters prove human pathogens.

The process of secretion in the Type $V$ secretion system

There are three mechanisms of Type V secretion system (T5SS). The bacterial protein prototypes secreted by T5SS (and dubbed sub class version of T5aSS) including an $\mathrm{N}$-terminal domain pathway as $40 \mathrm{Kd}$ to $400 \mathrm{Kd}$ and a protected C-terminal domain which constitutes a beta chamber $^{36}$.

In partner system, discharge path is divided into two parts

TpsA domain included as foreign proteins and leads to transferring folded proteins and $T p s B$ which is the maker of channel. TpsA and TpsB have different names in various bacteria.

\section{General discharge process is as follows}

Proteins are synthesized with an $\mathrm{N}$ terminal signal peptide that is directly transmitted to periplasm through the mechanism of Sec. Beta chamber can be imported into the outer membrane which is required to transport passenger domain to the extracellular space. In some cases, such as adhesions, the passenger domains attached to the beta container and proteins in the outer membrane remains firmly.

In other cases, the passenger domain is separated from beta chamber and forms a hydrolytic enzyme or toxin dissolved in it. Accordingly, these proteins are called automatic transporters that Cterminal domains form an aperture from a beta chamber through which $\mathrm{N}$-terminal domain can pass. However, further details in relation to recent structural studies show that the chamber cannot transport the passenger domain and needs auxiliary proteins.

For example, a helpful protein such as Omp85/YaeT facilitates transmission through the outer membrane. Subclass domain of the secretion of proteins through Type V secretion system (T5SS), are trimetric proteins that are formed from a beta chamber. The third type of proteins T5SS are T5bSS that include a pair of proteins that secrete the proteins inside this chamber of domain shared carries of beta chamber and other carrier proteins, secrete the proteins. This process is known as Two-Partner Secretion (TPS) system ${ }^{1}$, 22,36 .

A large number of proteins are secreted through T5SS (even more than T2SS). Only in T5aSS class, there are more than 500 proteins in the secretion list. The majority of proteins secreted by T5SS are human and animal pathogens. Proteins secreted by the T5SS include Adhesions like AIDAI and Ag43 related to E. coli, Hia in Haemophilus influenzae, YadA in Yersinia enteroliticola and Prn in Bordetella pertussis, toxins such as VacA in H.pylori, proteases such as IgA in Neisseria gonorrheae and Neisseria meningitides,sepA in shigella flexneri and PrtS in Serratia marcescens, and S-Layer proteins such as rOmpB in Rickettsia and Hsr in H.pylori. Also T5bSS (TPS) in the secretion of adhesion proteins in plant pathogens such as $\mathrm{Hec} A / \mathrm{HecB}$ in bacteria Dadantii dickeya and cytolysins like ShlA/ShlBin Serratia marcescens, HpmA/HpmB in Proteus Mirabilis and EthA/EthB in Edwardsiella tarda play an important role ${ }^{1,22,35,36}$

\section{Type VI Secretion system (T6SS)}

It was recently found that in most proteobacteria which are closely related to eukaryotic cells, there is a special secretion system. This secretion system has low number of genes, i.e., around 12-20 genes, usually found in pathogenicity islands and regulating the expression of these genes is usually done when in contact with the host cell. T6SS was firstly considered as a part of the pathogenetic islands in Gram-negative bacteria. But in 2006, it was introduced as secretion coding system ${ }^{31-32}$. Type VI secretion system (T6SS) is a system that can directly deliver proteins (single step) to the cytoplasm of host cells like T3SS and T4SS methods. More than a quarter of bacterial genomes, were sequenced including genes for T6SS components, which are mostly located in proteobacteria. But they can also be found in acidobacteria and planctomycetes ${ }^{1}$. In human and animal pathogens, such as Vibrio cholerae, Edwardsiella tarda, Pseudomonas aeruginosa, Francisella tularensis, and Burkholderia mallei, Type VI secretion system is present and in pathogen plants such as Agrobacterium tumefaciens, Pectobacterium atrosepticum and Xanthomonas oryzae, T6SS is required for the pathogenicity of 
the bacteria. Additionally, to transport and fix nitrogen in plants, Mesorhizobium loti and Rhizobium leguminosarum are also required. T6SS coding genes are also found in some nonsymbionts such as Myxococcus xanthus, Dechloromonas aromatica and Rhodopirellula baltica, and it is possible to form biofilm to comply with condition ${ }^{37}$.

\section{Components of Type VI secretion system}

T6SS system is composed of a minimum set of 13 units that are the core of the system. The core components have been named as TssA-TssM. However, they have a lot of generic names that are widely used (e.g., $H c p$ and $C l p V)^{38,39}$.

In addition to the core Tss genes, gene clusters T6SS may also include other components that are fixed in most T6SSs, e.g., structural component $F$ ha, proteins regulating PpkA and TagF pre-conversion and actuator of converting the dependent version to s54 $\mathrm{VasH}^{40}$. These clusters may show system specific substrates and protective or regulatory proteins. Various genomicanalysesindicate that T6SSs are almost present in a quarter of the bacterial genomes of sequencing and have extended among proteobacteria (except epsilon proteobacteria) and at least one third of the bacteria have several T6SSs, and this amount is more than 6 times in Yersinia pestis, Burkholderiapseudomallei ${ }^{37-38}$.These analyses show that T6SSs can be classified to 4 families or phylogenetic groups A, B, C, $\mathrm{D}^{1,6}$ that are corresponding to the I, II, V,IV ${ }^{37}$, although, a complete structure is not provided for T6SS subcompound like available structures for T3SS and $\mathrm{T}_{4} \mathrm{SS}^{38}$. It has recently been reported that the antibacterial T6SSs have been invented and effectively used to fight bacterial cells ${ }^{38,39}$.

\section{Anti eukaryotic T6SSs andthe role of Type VI secretion system in bacterial virulence}

It has been proved that T6SSs are necessary in virulence and/or normal reactions with eukaryotic cells in different bacteria especially pathogen bacteria ${ }^{37-38}$. Different bacteria are included a wide variety of species from plant pathogens (such as Pectobacterium atrosepticum) to animal pathogens (such as $E$. tarda and Salmonella Gallinarum) and even wellrecognized and important human pathogens (such as V. cholerae, Burkholderiasp and $P$. aeruginosa). However, the T6SS-mediated virulence principles are not known yet ${ }^{1,6}$.

The best studied system was investigated through Type- $\mathrm{V}$ secretion system in $V$. cholerae bacteria. One of the three $\operatorname{VgrG}$ homologues is associated-bacteria with T6SS in $V$. cholerae. $\operatorname{VgrG}-1$ is developed form of $\operatorname{VgrG}$ that comes with a C-terminal actin cross-linking domain with (ACD) that can polymerize G-actins monomers ${ }^{40}$. Transfer of $V g r G-1$ ACD through T6SS within mammals' macrophages has been proven that this transfer results in polymerization of host cells actins and subsequently cytotoxicity ${ }^{41-}$ ${ }^{42}$. In fact, ACD resulting from VgrG-1 can be considered an appropriate agent, because it does not need the T6SS secretion activity, and only requires the cytotoxic/cross-linking performance ${ }^{41}$. In the meanwhile, theVgrG-1 AC activity impedes phagocytosis process ${ }^{42}$. In fact, the inflammatory response of the host immune system to T6SS may intensify survival or $V$. cholerae in the intestine ${ }^{42}$.

Three related organisms (genetically) are B. mallei, B. pseudomalleiand B. thailandensis and all have T6SS (over four to six), which are essential for bacterial virulence. Severe human illnesses of Glanders and melioidosisare first created by the two organism ${ }^{39-41}$.

Burkholderia cenocepacia is an intracellular pathogen that leads to respiratory infections in patients with cystic fibrosis. A T6SS has been identified in this organism which is required for virulence. And although nothing secreted from this T6SS is still determined but it shows molecular results of T6SS activity in eukaryotic cell. Burkholderia cenocepacia survives inside the host cell macrophages within the vacuoles bondable to the membrane ( $B$. cenocepacia-containing vacuole, $B c C V)^{38-44}$.

\section{Antibacterial role for T6SSs}

Although most T6SSsplay an important role in the virulence of bacteria and / or reaction with eukaryotic cells, but some T6SSs also don't have such a role. Much similarity between the T6SS sectors and cell-puncturing part of bacteriophage increase this likelihood that T6SSs may be able to attack bacterial cells. In fact, for the first time T6SS antibacterial activity for T6SS has been demonstrated in $P$. aeruginosa bacteria ${ }^{43}$.

Then antibacterial T6SS were reported in a limited number of sectors associated with other bacteria such as V. cholerae, B. thailandensis and 
S. marcescens. These systems enable the bacteria to kill or inhibit the growth of rival bacteria.

\section{Type 7 secretion systems}

Although Gram-negative bacteria have only one membrane, but some of them and most mycobacteria have a cell wall, which are widely surrounded by lipids called mycomembrane and are mostly formed of mycolic acids covalently attached to free fats (glycols) ${ }^{44-48}$. As a result, the genomes of these species, code specific secretion systems that are named Type VII secretion systems (T7SS), to transfer virulence factors in their complex cell coverings. Sequencing of Mycobacterium bovis used in making BCG vaccine, as well as analysis of mutation in cluster the $E S X-1$ secretion in bacteria $M$. tuberculosis, confirms the hypothesis of a new system called Type VII secretion system ${ }^{45}$. Mycobacterium genomes have more than five T7SS gene clusters that do not complement each other in terms of performance and named as $E S X-1$ to $E S X$-5.T7SS systems for virulence of Staphylococcus aurous are necessary but it is not so in Listeria monocytogenes ${ }^{45,48}$.

Moreover, $E S X-1$ is required for virulence of fish Mycobacterium marinum pathogen and for conjugationprocess of non-pathogen bacteria such as Mycobacterium smegmatis.

Another T7S system they has recently been investigated is $E S X$-5. It is interesting that $E S X$-5 is the mostly recent T7S system discovered and is limited to mycobacterial species with slow growth that cover most pathogens such as $M$. tuberculosis, Mycobacterium leprae, Mycobacterium ulcerans and Mycobacterium marinum ${ }^{45,46}$. In M.marinum, ESX-5, the release of a large number of proteins is responsible for mycobacterial proteins called $P E$ and $P P E^{46}$, and the immune system play a role in the changes ${ }^{45}$. In Mycobacterium marinum, ESX-5 is responsible for the release of a large number of proteins in mycobacterial sections called PE and PPE proteins and play an important role modulation of immune system $^{45,47,48}$. While non-pathogenic mycobacteria only have a few of these proteins. But in pathogenic species, many proteins are expanded ${ }^{49}$.

\section{CONCLUSIONS}

This review paper presented an overview of components and seven bacterial secretion systems in pathogenic and nonpathogenic bacteria. Despite significant progress in understanding the performance of these systems which was achieved with the development of molecular diagnostic tools, the nature of some of the ingredients and molecular processes is still unknown. Thus, the importance of understanding these systems and their performance in recognizing bacteria, particularly pathogenic bacteria can create a new perspective to deal with the pathogens and developing new pharmaceutical compounds with more effective and targeted effects.

\section{ACKNOWLEDGEMENTS}

Thanks to Professor Afra Khosravi and Professor Nourekhoda Sadeghifard for their guidance. We also thank the Ilam University of Medical Sciences for their cooperation in the use of electronic resources.

\section{REFERENCES}

1. Tseng TT, Tyler BM and Setubal CJ. Protein secretion systems in bacterial-host associations, and their description in the gene onthology. BMC Microbiology. 2009; 9(1): 1-9.Doi:10.1186/14712180-9-S1-S2.

2. Kanonenberg K, Schwarz C and Schmitt L. Type I secretion system- a story of appendices. Research in Microbiology. 2013; 164: 596-604.

3. Economou A, Christie PJ, Fernandez RC, Palmer T, Plano GV, Pugsley AP. Secretion by numbers: protein traffic in prokaryotes. MolMicrobiol. 2006; 62: 308-319.

4. Lloubes R, Bernadac A, Houot L, Pommier S. Non classical secretion systems. Research in Microbiology 2013; 164: 655-663.

5. Natale P, Brüser T,Driessen A. Sec and Tat mediated protein secretion across the bacterial cytoplasmic membrane- distinct- translocases and mechanisms. BiochimicaetBiophysicaActa. 2008; 1778: 1735-1756.

6. Pallen M, Chaudhuri R and Henderson I. Genomic analysis of secretion systems. Current Opinion in Microbiology. 2003; 6(5): 519-527.

7. Wille T, Wagner C, Mittelstadt W, Blank K, Sommer E, Malengo G, Duhler D, et al. SiiA and SiiB are novel type I secretion system sub unite controlling SP-14 mediated adhesion of Salmonella enteric. Cellular Microbiology. 2014; 16(2): 161-178.

8. Bleves S, Viarre V, Salacha R, Michel GP, Filloux 
A, Voulhoux R. Protein secretion systems in Pseudomonas aeruginosa: a wealth of pathogenic weapons. Int J Med Microbiol. 2010; 300: 534543.

9. DelepelaireP.Type I secretion in gram-negative bacteria. Biochimica et Biophysica Acta. 2004; 1694(1-3): 149-161.

10. Holland IB, Schmitt L, Young J: Type 1 protein secretion in bacteria, the ABC- transporter dependent pathway (review). Molecular Membrane Biology. 2005; 22(1-2):29-39.

11. Harley KT, Djordjevic GM, Tseng TT and Saier $\mathrm{MH}$. Membrane fusion protein homologues in gram positive bacteria. MolMicrobiol. 2000; 36(2):516-517.

12. Binet $\mathrm{R}$ and Wandersman C. Cloning of the Serratia marcescens HasF gene encoding the Has ABC exporter outer membrane component: a TolC analogue. Mol Microbiol. 1996; 22: 265273.

13. Koronakis V, Sharff A, Koronakis E, Luisi B and Hughes C. Crystal structure of the bacterial membrane protein TolC central to multidrug efflux and protein export .Nature. 2000; 405: 914-919.

14. Gilson L, Mahanty HK, Kolter R. Four plasmid genes are required for colicin $\mathrm{V}$ synthesis, export, and immunity. J. Bacteriol. 1987; 169: 24662470.

15. Nakashima R, Sakurai, K, Yamasaki S, Nishino K, Yamaguchi A. Structures of the multidrug exporter AcrB reveal a proximal multisite drugbinding pocket. Nature. 2011; 480: 565-569.

16. Brandon L D, Goehring A, Janakiraman AW, Yan T, Wu J, Beckwith MB. IcsA, a polar localized auto transporter with an atypical signal peptide, uses the Sec apparatus for secretion although the Sec apparatus is circumferentially distributed. Mol Microbiol. 2003; 50:45-60.

17. Dalbey R and Kuhn A. Protein Trafûc in Gramnegative bacteria-how export and secretion proteins and their way. FEMS Microbiol Rev. 2012; 36: 1023-1045.

18. Desvaux M, Parham N J, Scott-Tucker A and Henderson IR. The general secretary pathway: a general misnomer. Trends in Microbiology. 2004; 12(7): 306-309.

19. Mole B M, Baltrus D A, Dangl JL and Grant S R. Global regulation networks in pathogenic bacteria. Trends Microbiol. 2007, doi:10.1016/ j.tim.2007.06.005.

20. Cianciotto N P. Type II secretion: a protein system for all seasons. Trends in Microbiology. 2005; 13(12): 581-588.

21. Voulhoux, R. Involvement of the twin-arginine translocation system in protein secretion via the type II pathway. EMBO J. 2001; 20: 67356741.

22. Henderson IR, Navaro-Garcia F, Desvaux M and Fernandez RC. Type V protein secretion pathway: the auto transporter story. Microbiol and Molecul BioloRevi. 2004; 68(4): 692-744.

23. Sandkvist M. Biology of type II secretion. Mol. Microbio. 2001; 40: 271-283.

24. Sandkvist M. Type II secretion and pathogenesis. Infect Immun 2001; 69: 35233535.

25. Soderberg MA. The type II protein secretion system of Legionella pneumophila promotes growth at low temperatures. J Bacteriol. 2004; 186: 3712-3720.

26. Koebnik R, Locher KP, and Van Gelder P. Structure and function of bacterial outer membrane proteins: barrels in a nutshell. $\mathrm{Mol}$ Microbiol. 2000; 37:239-253.

27. Cornelis GR. The type III secretion injectisome. Nat Rev Microbiol. 2006; 4(11): 823-825.

28. Grant SR, Fisher EJ, Chang JH, Mole BM, Dangl JL. Subterfuge and manipulation: type III effectors proteins of phytopathogenic bacteria. Annu Rev Microbiol. 2006; 60:425-449.

29. Tang X, Xiao Y and Zhou J M. Regulation of the type III secretion system in pathogenic bacteria. MPMI. 2006; 19(11): 1159-1166. Doi: 10.1094/ MPMI -19-1159.

30. Mota LJ, Cornelis GR. The bacterial injectisom kit: type III secretion system. Annals of Medicine. 2005; 37(4):234-249.

31. Desvaux M, Hebraud M, Talon R and Henderson I R. Secretion and subcellular localization of bacterial proteins: a semantic awareness issue. Trends in Microbiology 2009; 17(4): 139-145.

32. Silverman J M, Brunet R Y, Cascales E and Mogous J D. Structure and Regulation of the Type VI Secretion System. Annu Rev Microbiol. 2012; 66: 453-472. doi: 10.1146 annurev-micro121809-151619.

33. Fischer W, Haas R and Odenbreit S. Type IV secretion systems in pathogenic bacteria. Int $J$ Med Microbiol. 2002; 292: 159-168.

34. Culthurst S J. The type VI secretion system- a widespread and versatile cell targeting system. Research in Microbiology. 2013; 164: 640-654.

35. Juhas M. Type IV secretion systems and genomic islands-mediated horizontal gene transfer in Pseudomonas and Hemophilus. Microbiological Research. 2015; 170: 10-17.

36. Christie PJ, Whitaker N, Gonzales-Rivera C. The mechanism and structure of the bacterial type IV seceretion system. Biochim Biophys Acta. 2014; 1843(8):1578-1591.

37. Leo J C, Grin I and Link D. Type V secretion: 
mechanism(s) of auto transport through the bacterial outer memberan. Phil Trans R Soc B. 2012; 367: 1088- 1101.

38. Bhatty M, Laverdy Gomez JA, Christia PJ. The expending bacterial type VI secretion lexicon. Res Microbiol. 2013; 164(6):620-639.

39. Bingle L, Bailey C M and Pallen M J. Type VI secretion: a beginner's guide. Current Opinion in Microbiology. 2008; 11:3-8.

40. Boyer F, Fichant G, Berthod J, Vandenbrouck Y, Attree, I. Dissecting the bacterial type VI secretion system by a genome wide in silicoanalysis: what can be learned from available microbial genomic resources?. BMC Genomics. 2009; 100:104.

41. Pukatzki S, Ma AT, Sturtevant D, Krastins B, Sarracino D. Identiûcation of a conserved bacterial protein secretion system in Vibrio cholera using theDictyosteliumhost model system. Proc Natl Acad Sci. 2006; 103: $1528 \mathrm{e} 1533$.

42. Ma AT, McAuley S, Pukatzki S, Mekalanos JJ. Translocation of a Vibrio choleratype VI secretion effectors requires bacterial endocytosis by host cells. Cell Host Microbe. 2009; 5: 234243.

43. Hood RD, Singh P, Hsu F, Guvener T, Carl MA, Trinidad R. A type VI secretion system of
Pseudomonas aeruginosa targets a toxin to bacteria. Cell Host Microbe. 2010; 725-737.

44. Houben E, Bestebroer J, Ummels R, Wilson L, Piersma S, Jiménez C, et al. Composition of Type VII secretion system memberan complex. Molecular Microbiology. 2012; 86(2): 472-484.

45. Luirink J, Vandenbroucke-Grauls CM. Type VII secretion-mycobacteria show the way. Nat Rev Microbiol. 2007; 5: 883-891.

46. Abdallah AM, Verboom T, Weerdenburg EM, Gey van Pittius N, Mahasha PW, Jimenez C. PPE and PE_PGRS proteins of Mycobacterium marinum are transported via the type VII secretion system ESX-5. Mol Microbiol. 2008; 73: 329-340.

47. Weerdenburg EM, Abdallah AM, Mitra S, de Punder K. ESX-5 dificient in Mycobacterium marinumis hypervirulent in adult zebraûsh. Cell Microbiol 2012; 14: 728-739.

48. Binet R, Lettofe S, Ghigo JM, Delepelaire P and Wandersman C. Protein secretion by Gramnegative bacteria $\mathrm{ABC}$ exporters - a review. Gene. 1997; 192:7-11.

49. Terpe K. Overview of bacterial expression systems for heterologus protein production: from molecular and biochemical fundamental to commercial system. App Microbiol Biotechnol. 2006; 72: 211-222. 